\title{
Study of $p$-Layer Doping Density and Surface Band Bending on the Indium Tin Oxide/Hydrogenated Amorphous Silicon Heterojunction Solar Cells
}

\author{
D. RACHED ${ }^{a, *}$ AND H. MADANI YSSAD ${ }^{b}$ \\ ${ }^{a}$ Laboratoire de Physique des Plasmas, Matériaux Conducteurs et leurs Applications, \\ U.S.T.O.M.B. B.P.1505, El M'nouar, Oran, Algérie \\ ${ }^{b}$ Laboratoire LTE Département de Génie Mécanique, E.N.S.E.T B.P.1523, Route d'Es Senia, 31000 Oran, Algérie \\ (Received October 16, 2013; in final form June 20, 2014) \\ A solar cell (indium tin oxide (ITO) $/ p$-doped amorphous silicon $(p$-a-Si:H)/intrinsic polymorphous silicon \\ (i-pm-Si:H) $/ n$-doped crystalline silicon ( $n$-c-Si)) simulation, focused on $p$-layer doping density $N A$ and surface \\ band bending $E_{s b b}$ at the interface ITO/p-layer has been performed. Despite the deterioration of $p$-layer material \\ quality with doping density, the reduced bulk recombination was found to compensate for the increased loss in \\ the $p$-layer. An increase of $p$-layer doping density $N A$ and contact barrier height $\varphi_{b 0}$ (variation of the surface band \\ bending $E_{s b b}$ ) leads to an increase of the efficiency of heterojunction with intrinsic thin layer solar cells.
}

DOI: 10.12693 /APhysPolA.127.767

PACS: 73.61.Jc, 71.20.Mq, 88.40.hj, 88.40.jj

\section{Introduction}

Recently, heterojunction with intrinsic thin layer solar cell, were under intensive investigation since they are combining the low cost and low temperature process of hydrogenation amorphous silicon (a-Si:H) deposition coupled with the high stability of crystalline silicone (c-Si). An important scientific and technological progress on heterojunction with intrinsic thin layer (HIT) has led to solar cells with efficiencies up to $21 \%[1,2]$. Computing modeling of HIT structures has been carried out to understand carrier transport in these structures.

The parameters studied in this work include the $p$-layer doping density $N A$ and contact barrier height $\varphi_{b 0}$. The combination of these parameters determinates the built-in potential in $p-i-n$ and HIT solar cell. Simulation of a range of experimental results on HIT cells developed by Sanyo group and available in the literature [3] has been undertaken to extract typical parameters that characterize state-of-the-art HIT cells on $n$-type crystalline silicon substrates. The principal parameters used in this study are summarized in Table.

Experimentally if we interpose thin palladium or chrome film between transparent conductive oxides (TCO) and $p$-doped a-Si:H interface, it is possible to change the work function without a decrease of optical transmission $[4,5]$. With a change of the work function of the TCO, it is this $\varphi_{b 0}$ that varies.

In order to simulate the effects of $p$-layer doping density $N A$ and the height of the front contact barrier $\varphi_{b 0}$ in the structure HIT: ITO/p-a-Si:H/i-pm-Si:H/n-c-Si/Al, we have varied $N A$ and $\varphi_{b 0}$ to study the dependence

* corresponding author; e-mail: djaaffar31@yahoo.fr
TABLE

Principal input parameters.

\begin{tabular}{l|c|c|c}
\hline \hline \multicolumn{1}{c|}{ Parameters } & $p$-a-Si:H & i-pm-Si:H & $n$-c-Si \\
\hline$d\left[\mathrm{~A}^{\circ}\right]$ & 110 & 30 & $300 \times 10^{4}$ \\
$\chi[\mathrm{eV}]$ & 3.90 & 3.95 & 4.22 \\
$E_{\mu}[\mathrm{eV}]$ & 1.90 & 1.96 & 1.12 \\
$E_{a c}[\mathrm{eV}]$ & 0.27 & 0.92 & 0.06 \\
$\mathrm{ND}, N A_{\text {TOT }}\left[\mathrm{cm}^{-3}\right]$ & $1.0 \times 10^{19}$ & $1.0 \times 10^{14}$ & $3.0 \times 10^{11}$ \\
$E_{D}[\mathrm{eV}]$ & 0.050 & 0.050 & 0.005 \\
$E_{A}[\mathrm{eV}]$ & 0.030 & 0.030 & 0.003 \\
$G_{D 0}, \mathrm{G}_{A 0}\left[\mathrm{~cm}^{-3} \mathrm{eV}^{-1}\right]$ & $4.0 \times 10^{21}$ & $4.0 \times 10^{21}$ & $1.0 \times 10^{20}$ \\
$\mu_{\mathrm{e}-}\left[\mathrm{cm}^{2} / \mathrm{V} \mathrm{s}\right]$ & 20 & 30 & 1000 \\
$\mu_{\mathrm{h}+}\left[\mathrm{cm}^{2} / \mathrm{V} \mathrm{s}\right]$ & 4 & 12 & 450 \\
$\mathrm{NC}, \mathrm{NV}$ & $1 \times 10^{19}$ & $2 \times 10^{20}$ & $5.0 \times 10^{18}$ \\
$\sigma_{n}(\mathrm{tails})\left[\mathrm{cm}^{2}\right]$ & $10^{-17}$ & $10^{-17}$ & $2 \times 10^{-19}$ \\
$\sigma_{c}(\mathrm{tails})\left[\mathrm{cm}^{2}\right]$ & $10^{-16}$ & $10^{-15}$ & $2 \times 10^{-18}$ \\
$\sigma_{n}(\mathrm{midgap})\left[\mathrm{cm}^{2}\right]$ & $10^{-16}$ & $10^{-16}$ & $10^{-16}$ \\
$\sigma_{c}(\mathrm{midgap})\left[\mathrm{cm}^{2}\right]$ & $10^{-14}$ & $10^{-14}$ & $10^{-14}$ \\
$\varphi_{b 0}[\mathrm{eV}]$ & \multicolumn{3}{|c}{} \\
$\varphi_{b L}[\mathrm{eV}]$ & & $1.2-1.5$ & 0.06 \\
\end{tabular}

of the solar cell output.

The back contact barrier height $\varphi_{b L}$ is assumed to give neutral (no band bending) contact to the $\mathrm{n}^{+}$back layer. We chose the pm-Si:H as an intrinsic thin layer because of its excellent electric properties [6].

\section{Simulation model}

We have used amorphous semiconductor device modeling program (ASDMP) simulation model developed by the group of professor Chatterjee [7]. Professor Roca's group at École polytechnique de Paris, France, has demonstrated experimentally that ASDMP model mimics the performance of $p-i-n$ and HIT solar [8].

ASDMP examines the behaviour of semiconductor device structures under steady state in one dimension by 
solving simultaneously Poisson's equation (Eq. (1)), the continuity equations for free electrons and free holes (Eq. (2), (3)) using finite differences and the NewtonRaphson technique, and yields the $J(V)$ characteristics and the quantum efficiency. These equations are

$$
\begin{aligned}
& \frac{\mathrm{d}}{\mathrm{d} x}\left(\varepsilon(x) \frac{\mathrm{d} \psi(x)}{\mathrm{d} x}\right)=\rho(x), \\
& 0=\frac{1}{q} \frac{\mathrm{d} J_{n}(x)}{\mathrm{d} x}+G_{\text {opt }}(x)-R_{\text {net }}(x), \\
& 0=\frac{1}{q} \frac{\mathrm{d} J_{p}(x)}{\mathrm{d} x}+G_{\text {opt }}(x)-R_{\text {net }}(x),
\end{aligned}
$$

where $\varepsilon(x)$ is the dielectric permittivity of the semiconductor. $\Psi(x)$ is the potential energy of an electron at the vacuum level in electron volts, and $\rho(x)$ is the space charge density in the semiconductor. $J_{n}(x)$ and $J_{p}(x)$ are the electron and hole current, respectively, and $q$ is the charge of electron. The term $G_{\text {net }}(x)$ represents the net optical generation of free electron-hole pairs per unit volume, while $R_{\text {net }}(x)$ denotes the net recombination of free carriers per unit volume. The boundary conditions used for the Poisson equation are

$$
\psi(0)=\varphi_{b 0}+\chi_{0}-\varphi_{b L}-\chi_{L}-V
$$

and

$$
\psi(L)=0
$$

where $\Psi(0)[\Psi(L)]$ is the vacuum level at $x=(L)$, $\varphi_{b 0}\left(\varphi_{b L}\right)$ the front (back) contact barrier height and $\chi_{0}$ $\left(\chi_{L}\right)$ the electron affinity of the material at $x=0(L)$.

$V$ is the applied forward biased potential. The typical gap-state distributions used in the calculations consists of U-shaped model, and two Gaussian distribution functions to simulate the dangling bond states. The generation term in the continuity equations has been calculated using a semiempirical model [9] that has been integrated into the modelling program. Both secular interference effects and diffused reflectances and transmittances due to interface roughness are taken into account.

\section{Results and discussion}

The structure used in this present work consists of a HIT structure ITO/p-a-Si:H/i-pm-Si:H/n-c-Si/Al, and is illustrated in Fig. 1. The principal parameters of each layer are summarized in Table. In these cells, the contact barrier heights for a cell with the $p$-layer in contact with a ITO at $x=0$ and the $n$-layer in contact with a metal at $x=L$, are given by

$$
\varphi_{b 0}=E_{\mu}(p)-E_{a c}(p)-E_{s b b}
$$

and

$$
\varphi_{b L}=E_{a c}(n),
$$

where $E_{\mu}(p)$ and $E_{a c}(p)$ represent respectively the mobility band gap and the activation energy of the $p$-layer, and $E_{s b b}$ is the surface band banding due to the Schottky barrier at the ITO $/ p$ interface.

Figure 2 shows the recombination under AM 1.5 light as a function of position for the $p$-layer doping density $N A=10^{19}$ and $2.8 \times 10^{19} \mathrm{~cm}^{-3}$. The reduced bulk

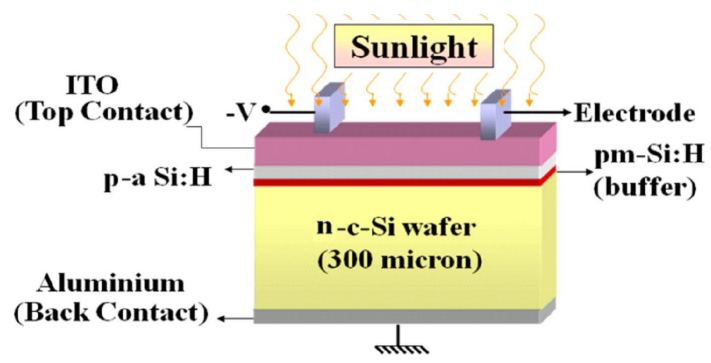

Fig. 1. Schematic diagram of a HIT $n$-type c-Si substrates (ITO $/ p$-a-Si:H $/ \mathrm{i}-\mathrm{pm}-\mathrm{Si}: \mathrm{H} / n-\mathrm{c}-\mathrm{Si} / \mathrm{Al}$ ) Solar Cell.

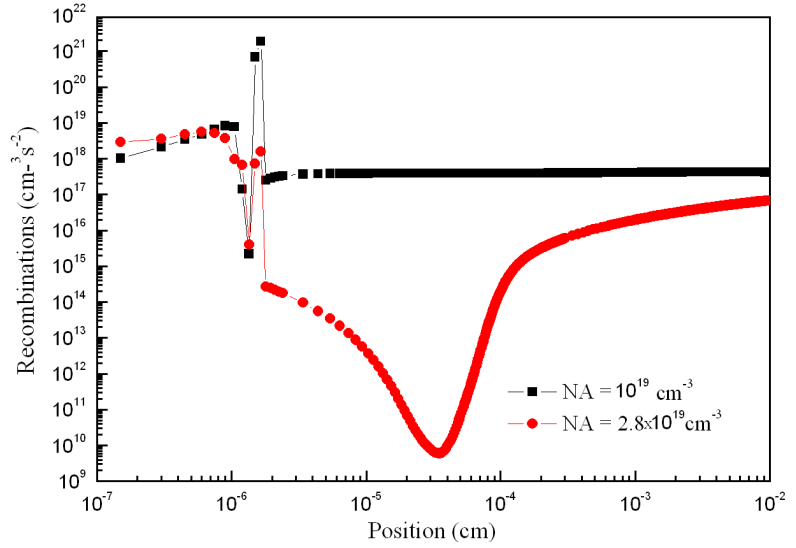

Fig. 2. The recombination under AM 1.5 light as a function of position for $N A=10^{19}$ and $2.8 \times 10^{19} \mathrm{~cm}^{-3}$ for the front contact height barrier $\phi_{b 0}=1.20 \mathrm{eV}$.

recombination was found when we increase the NA. This reduction of the recombination is due to the decrease of the electric field seen in Fig. 3. This decrease of the electric field over the depletion region let the photogenerated holes able to pass to the front contact and the electrons to the back $[10,11]$.

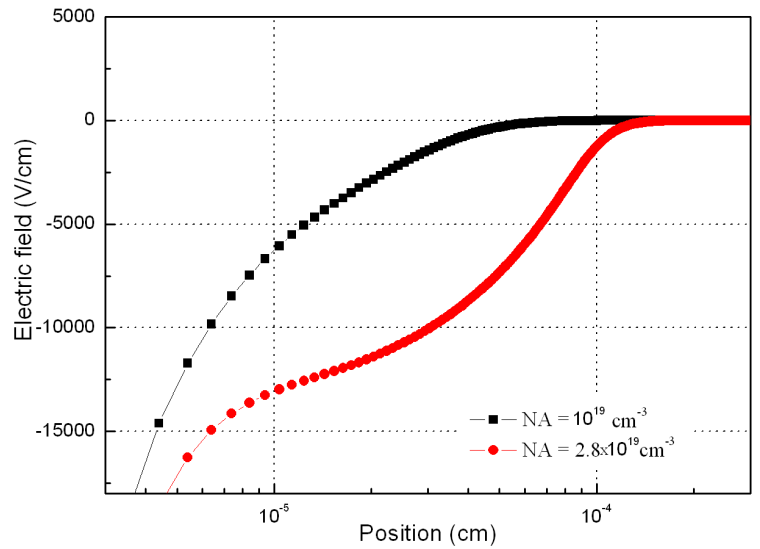

Fig. 3. The electric field as a function of $p$-layer doping density $N A=10^{19}$ and $2.8 \times 10^{19} \mathrm{~cm}^{-3}$ for the front contact height barrier $\phi_{b 0}=1.20 \mathrm{eV}$.

The sensitivity of the short circuit current $J_{\mathrm{sc}}$ (a), the open circuit voltage $V_{\mathrm{oc}}(\mathrm{b})$, the fill factor FF (c) and the cell efficiency $\eta(\mathrm{d})$ to the $p$-layer doping density $N A$ and front contact barrier height $\varphi_{b 0}$ is shown in Fig. 4. $\varphi_{b 0}$ is equal to 1.20 and $1.50 \mathrm{eV} . N A$ is equal to $10^{19}$ and 

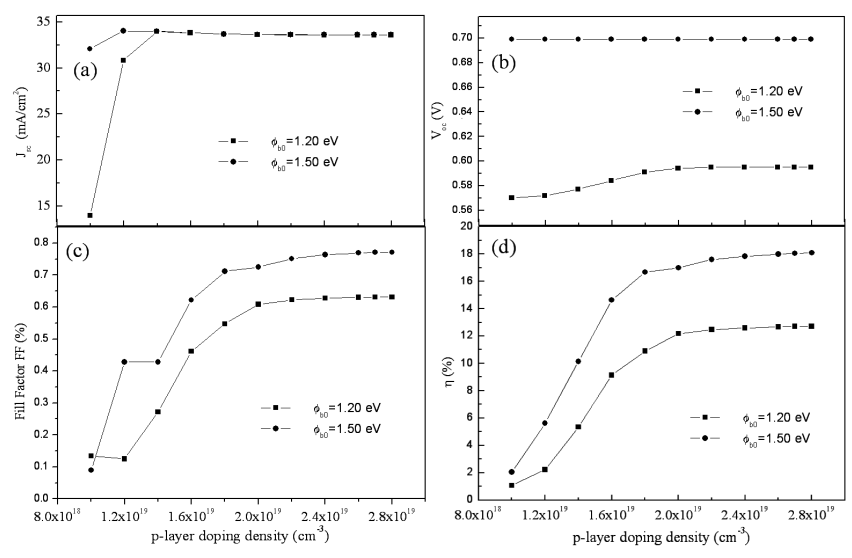

Fig. 4. Plot of (a) $V_{\mathrm{oc}}$, (b) FF, (c) $I_{\mathrm{sc}}$, and (d) $\eta$ vs. $p$ layer doping density for the front contact height barrier $\phi_{b 0}=1.20 \mathrm{eV}$ and $1.50 \mathrm{eV}$.

$2.8 \times 10^{19} \mathrm{~cm}^{-3}$. Despite the deterioration of $p$-layer material quality with doping density, the solar cell output parameters are sensitive to NA. A large improvement of FF has been obtained because of the reduced bulk recombination seen in Fig. 2.

Maximum sensitivity of all the solar cell output parameters became evident when we increase the front contact barrier height to $\varphi_{b 0}=1.50 \mathrm{eV}$.

Figure 5 shows that the built-in potential $V_{\mathrm{bi}}$ depends to the front contact barrier height $\varphi_{b 0}$. The increase of

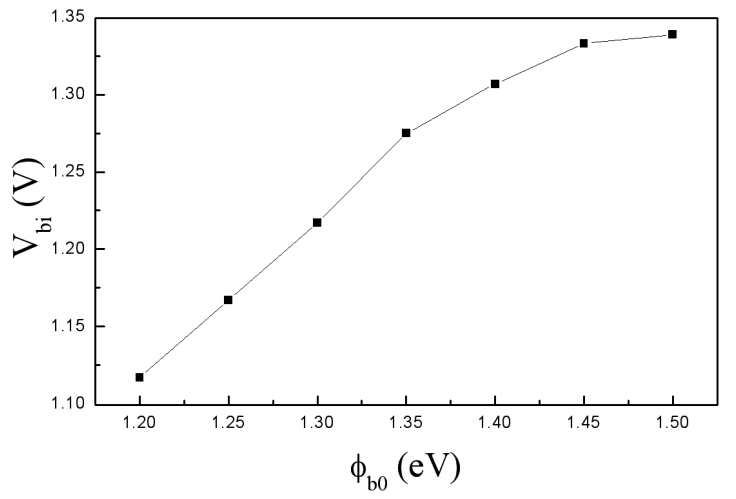

Fig. 5. The built-in potential $V_{\mathrm{bi}}$ vs. front contact barrier height for cells with $11 \mathrm{~nm}$ of $p$-layer.

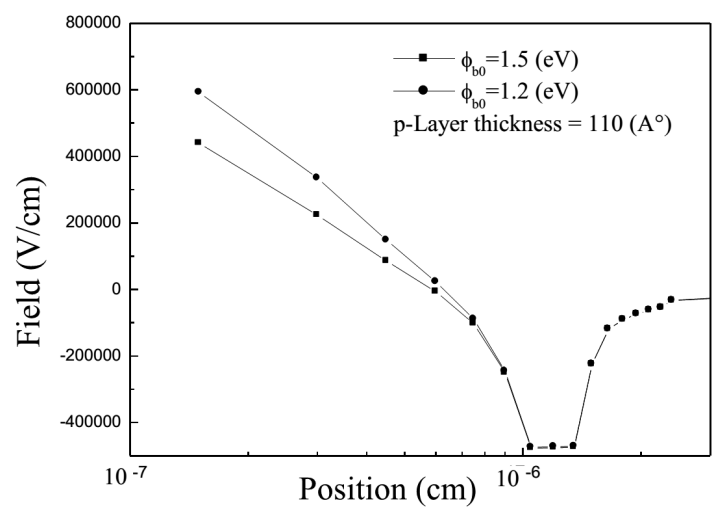

Fig. 6. The electric field vs. position for cells with $11 \mathrm{~nm}$ of $p$-layer and the front contact height barrier $\phi_{b 0}=1.50 \mathrm{eV}$ and $1.20 \mathrm{eV}$.
$V_{\mathrm{bi}}$ is the result of the change in the potential barrier which is reduced at the ITO $/ p$-layer. We notice in Fig. 5 a decrease of the electric field when we increase the $\varphi_{b 0}$ value. This decrease let the holes able to pass from the $p$-layer to the contact.

Figure 6 indicates that a remarkable improvement on $V_{\mathrm{oc}}$ and $\mathrm{FF}$ has been achieved with the improvement of $\varphi_{b 0}$. This augmentation is due to the reduction of the surface potential barrier. A reduction of this surface potential barrier leads to an augmentation of $V_{\mathrm{bi}}$ and consequently an increase of $V_{\mathrm{oc}}$. The reduction of the field at the ITO $/ p$-layer interface seen in Fig. 5 will support the passage of the holes towards ITO. A large improvement of $\mathrm{FF}$ has been obtained because of the reduced interface recombination as well as better ohmic contact between ITO and $p$-layer.

\section{Conclusion}

In conclusion, the amorphous HIT solar cells output parameters are sensitive to the doping density $N A$ and front contact barrier $\varphi_{b 0}$.

In spite of an increased loss in the $p$-doped a-Si:H when we increase the doping density a large improvement of FF has been obtained. The reduced bulk recombination was found to compensate for the increased loss in the $p$-layer.

An increase of front contact height barrier leads to an increase of the efficiency of solar cells. The explanation of an increased $V_{\mathrm{oc}}$ is attribute to an enhancement of the $V_{\mathrm{bi}}$ and an improvement of $\mathrm{FF}$ is due to the decrease of the electric field between ITO and $p$-a-Si:H.

\section{References}

[1] T. Mishima, M. Taguchi, H. Sakata, E. Maruyama, Sol. En. Mater. Sol. Cells 95, 18 (2011).

[2] L. Geerligs, I.G. Romijn, A. Burgers, N. Guillevin, A.W. Weeber, J.H. Bultman, H.F. Wang, F. Lang, W. Zhao, G. Li, Z. Hu, J.F. Xiong, A. Vlooswijk, in: Proc. 38th IEEE Photovoltaic Specialists Conf. (PVSC '12), 2012, p. 001701.

[3] M. Taguchi, E. Maruyama, M. Tanaka, Jpn. J. Appl. Phys. 47, 814 (2008).

[4] P. Roca y Cabarrocas, S. Ramprashad, J.Z. Liu, V. Chu, A. Maruyama, S. Wager, in: Proc. 21st IEEE Photovoltaic Conf., Kissimmee, Vol. 2, 1990, p. 1610.

[5] P. Roca y Cabarrocas, U. Eicker, in: Proc. Tenth European Photovoltaic Solar Energy Conf., Lisbon, 1991, p. 335.

[6] A. Fontcuberta, Ph.D. Thesis, Laboratoire LPICM, Ecole Polytechnique, France 2002.

[7] P. Nandita, Ph.D. Thesis, Energy Research Unit, Indian Association for the Cultivation of Science, Jadavpur, Calcutta, India 2000.

[8] Y. Poissant, P. Chatterjee, P. Roca y Cabarrocas, J. Appl. Phys. 93, 170 (2003).

[9] F. Leblanc, J. Perrin, J. Schmitt, J. Appl. Phys. 75, 1074 (1994).

[10] T. Desrues, Ph.D. Thesis, Institut National des Sciences Appliquées de Lyon, France 2009.

[11] L. Mai, Z. Hameiri, B.S. Tjahjono, S.R. Wenham, A. Sugianto, M.B. Edwards, in: Conference records of the 34th IEEE PVSC, Philadelphia (USA), 2009, p. 001811. 